\title{
Red and White Blood Cell Counts Are Associated With Bone Marrow Adipose Tissue, Bone Mineral Density, and Bone Microarchitecture in Premenopausal Women
}

\author{
Sai Polineni, ${ }^{1}$ (D) Megi Resulaj, ${ }^{1}$ Alexander T Faje, ${ }^{1,2}$ Erinne Meenaghan, ${ }^{1}$ Miriam A Bredella, ${ }^{2,3}$ \\ Mary Bouxsein, ${ }^{2,4}$ (1) Hang Lee, ${ }^{2,5}$ Ormond A MacDougald, ${ }^{6}$ Anne Klibanski, ${ }^{1,2}$ and Pouneh K Fazeli ${ }^{1,2,7}$ (i) \\ ${ }^{1}$ Neuroendocrine Unit, Massachusetts General Hospital, Boston, MA, USA \\ ${ }^{2}$ Harvard Medical School, Boston, MA, USA \\ ${ }^{3}$ Department of Radiology, Massachusetts General Hospital, Boston, MA, USA \\ ${ }^{4}$ Center for Advanced Orthopaedic Studies, Beth Israel Deaconess Medical Center, Boston, MA, USA \\ ${ }^{5}$ Biostatistics Center, Massachusetts General Hospital, Boston, MA, USA \\ ${ }^{6}$ Department of Molecular and Integrative Physiology, University of Michigan Medical School, Ann Arbor, MI, USA \\ ${ }^{7}$ Division of Endocrinology and Metabolism, University of Pittsburgh School of Medicine, Pittsburgh, PA, USA
}

\begin{abstract}
Bone marrow adipose tissue (BMAT) resides within the bone marrow microenvironment where its function remains poorly understood. BMAT is elevated in anorexia nervosa, a disease model of chronic starvation, despite depletion of other fat depots. In addition to BMAT, the marrow microenvironment also consists of osteoblast and hematopoietic progenitors. BMAT is inversely associated with bone mineral density (BMD) in multiple populations including women with anorexia nervosa, and regulates hematopoiesis in animal models. We hypothesized that BMAT would be associated with circulating populations of hematopoietic cells (red and white blood cells) in humans and performed a post hoc analysis of two studies-a cross-sectional study and a longitudinal study-to investigate this hypothesis. We studied 89 premenopausal women cross-sectionally (median age [interquartile range], 27 [24.5, 31.7] years), including 35 with anorexia nervosa. We investigated associations between red blood cell (RBC) and white blood cell (WBC) counts and BMAT assessed by ${ }^{1} \mathrm{H}$-magnetic resonance spectroscopy, BMD assessed by DXA, and bone microarchitecture assessed by HRPQCT. In addition, we analyzed longitudinal data in six premenopausal women with anorexia nervosa treated with transdermal estrogen for 6 months and measured changes in BMAT and blood cell counts during treatment. Cross-sectionally, BMAT was inversely associated with WBC and RBC counts. In contrast, BMD and parameters of bone microarchitecture were positively associated with WBC and RBC. In women with anorexia nervosa treated with transdermal estrogen for 6 months, decreases in BMAT were significantly associated with increases in both $\mathrm{RBC}$ and hematocrit (rho $=-0.83, p=0.04$ for both). In conclusion, we show that BMAT is inversely associated with WBC and RBC in premenopausal women, and there is a potential association between longitudinal changes in BMAT and changes in RBC. These associations warrant further study and may provide further insight into the role and function of this understudied adipose depot. ( 2020 American Society for Bone and Mineral Research.
\end{abstract}

KEY WORDS: ANOREXIA NERVOSA; BONE MICROARCHITECTURE; BONE MINERAL DENSITY; HEMATOPOIESIS; MARROW ADIPOSE TISSUE

\section{Introduction}

The he bone marrow consists of two types of stem cells: (i) mesenchymal stem cells, which differentiate into osteoblasts or adipocytes, and (ii) hematopoietic stem cells, which differentiate into red blood cells (RBCs), white blood cells (WBCs), or platelets. In murine models, osteoblasts are important regulators of hematopoiesis ${ }^{(1)}$ and induced osteoblast-depletion results in a decrease in erythroid, lymphoid, and myeloid progenitors. ${ }^{(2)}$ In humans, both RBC and WBC populations are associated with bone mineral density (BMD) and anemia has been shown to be an independent predictor of fracture. ${ }^{(3)}$ Further, low hemoglobin levels are associated with low BMD in older adults, ${ }^{(4)}$ and higher rates of bone loss at the hip have been associated with both an increased risk of anemia as well as low lymphocyte counts in men 65 years or older. ${ }^{(5)}$

Bone marrow adipose tissue (BMAT), another component of the bone marrow microenvironment, is also associated with bone parameters in humans. BMAT is inversely associated with BMD in populations of healthy adults ${ }^{(6,7)}$ and also in a model of

Received in original form August 29, 2019; revised form January 3, 2020; accepted February 13, 2020. Accepted manuscript online February $20,2020$. Address correspondence to: Pouneh K Fazeli, MD, MPH, Division of Endocrinology and Metabolism, University of Pittsburgh School of Medicine, 200 Lothrop Street, BST W1061 Pittsburgh, PA 15213, USA. E-mail: pkfazeli@pitt.edu

The peer review history for this article is available at https://publons.com/publon/10.1002/jbmr.3986.

Journal of Bone and Mineral Research, Vol. 35, No. 6, June 2020, pp 1031-1039.

DOI: $10.1002 / j b m r .3986$

() 2020 American Society for Bone and Mineral Research 
chronic starvation, anorexia nervosa. ${ }^{(8)}$ Women with anorexia nervosa have higher levels of BMAT compared to normal-weight controls despite having significantly lower levels of subcutaneous and visceral adipose tissue depots. ${ }^{\left({ }^{(8)}\right.}$ Although the function of BMAT is not currently known, the fact that this adipose tissue depot is preserved during chronic starvation, when other fat depots are being used as a source of energy, suggests it may have an important role.

In murine models, granulocyte-colony stimulating factor, which leads to an increase in circulating neutrophils, has been associated with reductions in $\mathrm{BMAT}^{(9)}$ and BMAT has also been associated with reduced hematopoiesis. ${ }^{(10)}$ Therefore, to better understand the potential function and determinants of BMAT in humans, we explored the association between BMAT and cells from the hematopoietic lineage, specifically $R B C$ and WBC counts in premenopausal women using both cross-sectional and longitudinal study designs. We hypothesized that BMAT would be negatively associated with cells from the hematopoietic lineage.

\section{Materials and Methods}

\section{Subjects}

We performed post hoc analyses of two prior studies. The first was a study investigating levels of BMAT in premenopausal women, inclusive of women with anorexia nervosa, normalweight controls, and obese women. ${ }^{(11-14)}$ The second study was a longitudinal study investigating the effects of low-dose, transdermal estrogen on BMD and BMAT in premenopausal women with anorexia nervosa. ${ }^{(15)}$ Eighty-nine premenopausal women were studied cross-sectionally, inclusive of 35 women with anorexia nervosa (median age [interquartile range], 26.8 $[24.6,31.0]$ years; range, 20.3 to 45.1 years). In addition, we studied six women with anorexia nervosa (median age [interquartile range], 34.3 [28.5, 45.4] years; range, 25.3 to 45.5 years) longitudinally for 6 months, during which time they were treated with low-dose, transdermal estrogen (Climara Pro; Bayer Pharmaceuticals, Whippany, NJ, USA) a treatment that has been associated with a reduction in BMAT in postmenopausal and premenopausal women. ${ }^{(15,16)}$ This particular transdermal formulation contains $0.045 \mathrm{mg}$ of estradiol/day and $0.015 \mathrm{mg}$ of levonorgestrel/ day, a progesterone, which is necessary for the prevention of endometrial hyperplasia in women with an intact uterus. As previously described, ${ }^{(15)}$ this formulation was chosen because it contains a dose of estradiol that we hypothesized would be effective in improving BMD based on data in postmenopausal women. ${ }^{(17)}$ BMAT and/or bone parameters from a subset of subjects were previously reported. ${ }^{(11-15,18)}$ Hematologic data have not been previously reported. Subjects were recruited either through online advertisements or through referrals from local eating disorder providers for both the cross-sectional and longitudinal studies. Subjects with anorexia nervosa met Diagnostic and Statistical Manual of Mental Disorders (DSM-5) criteria for the disorder. ${ }^{(19)}$ Subjects without anorexia nervosa had a $\mathrm{BMI}>18.5 \mathrm{~kg} / \mathrm{m}^{2}$ (range, 20.8 to $41.7 \mathrm{~kg} / \mathrm{m}^{2}$ ), none reported a past or current history of an eating disorder, none were taking medications known to affect bone mass, and all reported having regular menstrual cycles. Participants who had abnormal thyroid function tests or chronic diseases that affect BMD (other than anorexia nervosa) were excluded.

\section{Cross-sectional study}

All subjects were evaluated at the Translational and Clinical Research Center at the Massachusetts General Hospital during two study visits: a screening visit and a baseline study visit. All subjects had a complete blood count inclusive of RBC count, WBC count, hemoglobin and hematocrit levels, and a platelet count at the time of their screening visit, performed by a clinical laboratory (LabCorp, Burlington, NC, USA). Subjects then returned for a study visit within 3 months of their screening visit, at which time they were weighed on an electronic scale while wearing a hospital gown, their height was measured on a single stadiometer as the average of three readings, and radiologic imaging, including dual-energy X-ray absorptiometry (DXA), ${ }^{1} \mathrm{H}$-magnetic resonance spectroscopy ( $\left.{ }^{1} \mathrm{H}-\mathrm{MRS}\right)$, and highresolution peripheral quantitative $\mathrm{CT}$ (HR-pQCT), was performed (radiologic imaging is described below in the Radiologic Imaging section).

\section{Longitudinal study}

All subjects were evaluated at the Translational and Clinical Research Center at the Massachusetts General Hospital for baseline and 6-month study visits. After the baseline visit, subjects were started on low-dose, transdermal estrogen/progesterone and they continued using the patch until their 6-month study visit. At each study visit, all six subjects had a complete blood count, inclusive of RBC count, WBC count, hemoglobin and hematocrit levels, and a platelet count, and ${ }^{1} \mathrm{H}-\mathrm{MRS}$ (described in the ${ }^{1} \mathrm{H}$-MRS section below) performed.

The study complied with the Health Insurance Portability and Accountability Act guidelines and was approved by the Partners Institutional Review Board. All subjects provided written informed consent prior to the initiation of study procedures.

\section{Radiologic imaging}

\section{DXA}

All subjects underwent DXA to measure areal BMD of the posterior-anterior (PA) lumbar spine $\left(L_{1}-L_{4}\right)$, lateral spine $\left(L_{2}-L_{4}\right)$, left total hip, left femoral neck, and total body using a Hologic Discovery A densitometer (Hologic Inc., Bedford, MA, USA). Coefficients of variation (CVs) for the measurement of BMD by DXA have been reported to be less than $2.2 \% .^{(20)}$

\section{High resolution peripheral quantitative computed tomography} (HR-pQCT)

HR-pQCT (isotropic voxel size of $82 \mu \mathrm{m}^{3}$ ) was performed in the nondominant distal radius and tibia (Xtreme CT; Scanco Medical AG, Brüttisellen, Switzerland) as previously described. ${ }^{(21)}$ As previously described, ${ }^{(22,23)}$ linear micro-finite element analysis of HR-pQCT images was subsequently performed in order to estimate the biomechanical properties of the distal radius and the distal tibia under uniaxial compression loading. Outcomes from linear micro-finite element analysis included both compressive stiffness $(\mathrm{kN} / \mathrm{mm})$ and failure load $(\mathrm{kN})$. The biomechanical properties estimated from micro-finite element analysis are strongly correlated with those measured through the ex vivo testing of elderly human cadaveric radii. ${ }^{(24)}$ 
The Metabolic Imaging Core of the Nutrition and Obesity Research Center at Harvard performed ${ }^{1} \mathrm{H}-\mathrm{MRS}$ as previously described. ${ }^{(8)}$ Briefly, lipid content of the $L_{4}$ vertebra, proximal femoral epiphysis, metaphysis, and mid-diaphysis was measured using ${ }^{1} \mathrm{H}$-MRS (Siemens Trio, 3T; Siemens Medical Systems, Erlangen, Germany). This was performed by placing a voxel measuring $15 \times 15 \times 15 \mathrm{~mm}^{3}\left(3.4 \mathrm{~mL}\right.$ ) within the body of the $L_{4}$ vertebra and then using point-resolved spatially localized spectroscopy (PRESS) pulse sequence without water suppression (parameters: echo time [TE] of $30 \mathrm{~ms}$, repetition time [TR] of $3000 \mathrm{~ms}$, eight acquisitions, 1024 data points, and receiver bandwidth of $2000 \mathrm{~Hz}$ ), single-voxel ${ }^{1} \mathrm{H}-\mathrm{MRS}$ data were obtained. Similarly, in the femur, single-voxel ${ }^{1} \mathrm{H}-\mathrm{MRS}$, using the same nonwater suppressed PRESS pulse sequence, was performed in the femur after a $12 \times 12 \times 12 \mathrm{~mm}^{3}(1.7 \mathrm{~mL})$ voxel was placed in the proximal femoral epiphysis and repeated after voxel placement in the mid-diaphysis and the intertrochanteric region of the femoral metaphysis. Automated procedures were used to optimize gradient shimming and transmit and receive gain. We have reported that the CV for marrow fat quantification, obtained by scanning five subjects two times each, is $3 \%$. ${ }^{(25)}$

${ }^{1} \mathrm{H}$-MRS data were fitted using LCModel software (version 6.1-4A; Stephen Provencher, Oakville, ON, Canada). Metabolite quantification was obtained using eddy current correction and water scaling after data were transferred to a Linux workstation. A fitting algorithm customized for analysis of bone marrow was used to provide estimates for all of the lipid signals combined $(0.9,1.3$, and $2.3 \mathrm{ppm})$. The lipid estimates for bone marrow using LCModel software were automatically scaled to an unsuppressed water peak (4.7 ppm) and results were expressed as a lipid to water ratio.

\section{Statistical analysis}

JMP Pro 13.0 (SAS Institute, Inc., Cary, NC, USA) software was used to perform statistical analyses. The Student's $t$ test was used to compare means and standard deviations (SDs) unless data were not normally distributed, in which case the Wilcoxon test was used to compare median (interquartile range). Univariate associations were assessed using Pearson correlation coefficients $(R)$, or if data were non-normally distributed, Spearman coefficients (rho). Spearman coefficients were calculated in the analysis of the longitudinal data, given the small sample size $(n=6)$. A $p$ value $<0.05$ was used to indicate significance.

\section{Results}

\section{Clinical characteristics}

Clinical characteristics of the study subjects, including BMD and bone microarchitecture data, are listed in Table 1. Subjects were all premenopausal. Subjects in the cross-sectional study were a median of 27 years of age (median age [interquartile range], 27 [24.5, 31.7] years; range, 20.0 to 45.7 years). Subjects in the longitudinal study had anorexia nervosa for a median of 13 years (range, 3 to 17 years) and had amenorrhea for a median of 135 months (range, 34 to 204 months). BMAT was inversely associated with BMD, parameters of bone microarchitecture, and bone strength (Table 2).
BMAT at the vertebra and femur was inversely associated with WBC and/or RBC counts. In particular, BMAT at the $\mathrm{L}_{4}$ vertebra was significantly inversely associated with WBC count (rho $=-0.37, p=0.0003$ ) (Fig. $1 A$ and $B$ ). BMAT at the femoral diaphysis and femoral metaphysis were both significantly and inversely associated with WBC and RBC counts (Table 3 ). There was a trend toward an inverse association between BMAT at the femoral diaphysis and both hemoglobin and hematocrit levels ( $r$ o $=-0.20, p=0.057$ for both). There were no significant associations between BMAT and platelet count.

When we divided the group based on diagnosis/BMI: anorexia nervosa ( $n=35)$, normal-weight $(n=28)$, and overweight/obese $(n=26)$, there were no significant associations between BMAT and any hematologic parameters in the women with anorexia nervosa or normal-weight women. In the women who were overweight/obese, in contrast to what was observed in the group as a whole, there was a significant positive association between $\mathrm{L}_{4}$ vertebral BMAT and RBC count (rho $=0.43, p=0.03$ ) and between BMAT at the femoral metaphysis and WBC count (rho $=0.46, p=0.02$ ). There was also a positive association between BMAT at the femoral metaphysis and platelet count in this group $(R=0.48, p=0.01)$.

\section{Association between blood counts and bone parameters}

\section{$B M D$}

There were significant and positive associations between WBC and $\mathrm{RBC}$ counts and BMD at the spine and hip (Table 4). BMD at the PA spine was significantly associated with RBC count (rho $=0.32, p=0.002$ ) and WBC count (rho $=0.45, p<0.0001$ ). There were no significant associations between hemoglobin or hematocrit and BMD. Platelet count was positively associated with both total hip BMD $(R=0.21, p=0.04)$ and femoral neck $\operatorname{BMD}(R=0.21, p=0.04)$.

\section{Bone microarchitecture and estimated bone strength}

Parameters of bone microarchitecture and estimated bone strength (stiffness and failure load) at the radius and tibia were significantly associated with WBC and RBC counts (Table 4). WBC count was positively associated with estimates of failure load at the radius (rho $=0.40, p<0.0001$ ) and tibia ( $r$ o $=0.41$, $p<0.0001$ ) (Fig. $2 A-D$ ). RBC count was also positively associated with estimates of failure load at both the radius ( $r h o=0.26, p=$ 0.01 ) and tibia (rho $=0.24, p<0.03$ ). There were no significant associations between microarchitecture parameters and hemoglobin or hematocrit levels. Platelet count was only associated with trabecular bone volume fraction at the radius ( $r$ ho $=0.24$, $p<0.03$ ) and trabecular thickness at the radius (rho $=0.22$, $p<0.04)$.

\section{Association between changes in blood counts and changes in BMAT}

Treatment with transdermal estrogen is associated with changes in BMAT in both postmenopausal women and premenopausal women. ${ }^{(15,16)}$ In an exploratory analysis, we measured complete blood counts at baseline and after 6 months of treatment with transdermal estrogen in six amenorrheic women with anorexia nervosa. Changes in BMAT at the femoral diaphysis were significantly inversely associated with changes in red blood cell count over the 6-month study (Fig. $3 A$ and $B$ ) and with changes in 
Table 1. Clinical Characteristics of Participants in Cross-Sectional Study and Baseline Characteristics of Subjects With Anorexia Nervosa in Longitudinal Study

\begin{tabular}{|c|c|c|}
\hline Characteristic & Cross-sectional study participants $(n=89)$ & Longitudinal study participants $(n=6)$ \\
\hline Age (years) & $27.0(24.5,31.7) ;$ range, $20-45.7$ & $35.7 \pm 3.5($ range, $25.3-45.5)$ \\
\hline BMI $\left(\mathrm{kg} / \mathrm{m}^{2}\right)$ & $22.1(17.8,30.8)$ & $17.1 \pm 1.4$ \\
\hline \multicolumn{3}{|l|}{ BMD } \\
\hline Posterior-anterior spine BMD $\left(\mathrm{g} / \mathrm{cm}^{2}\right)$ & $0.955(0.854,1.078)$ & $0.808 \pm 0.111$ \\
\hline Lateral spine BMD $\left(\mathrm{g} / \mathrm{cm}^{2}\right)$ & $0.743 \pm 0.149$ & $0.628 \pm 0.109$ \\
\hline Total hip BMD $\left(\mathrm{g} / \mathrm{cm}^{2}\right)$ & $0.919 \pm 0.170$ & $0.739 \pm 0.093$ \\
\hline Femoral neck BMD $\left(\mathrm{g} / \mathrm{cm}^{2}\right)$ & $0.799 \pm 0.159$ & $0.647 \pm 0.087$ \\
\hline Total body BMD $\left(\mathrm{g} / \mathrm{cm}^{2}\right)$ & $1.020 \pm 0.097$ & $0.982 \pm 0.052$ \\
\hline \multicolumn{3}{|l|}{ Bone marrow adipose tissue } \\
\hline $\mathrm{L}_{4}$ vertebra (lipid/water) & $0.54(0.40,0.87)$ & $1.18 \pm 0.42$ \\
\hline Femoral epiphysis (lipid/water) & $7.25 \pm 2.90$ & $8.17 \pm 2.17$ \\
\hline Femoral diaphysis (lipid/water) & $4.84(2.61,7.09)$ & $7.58 \pm 1.71$ \\
\hline Femoral metaphysis (lipid/water) & $3.18(2.04,4.89)$ & $5.77 \pm 1.96$ \\
\hline \multicolumn{3}{|l|}{ Microarchitecture parameters at radius } \\
\hline Trabecular BV/TV (\%) & $0.128(0.109,0.151)$ & $0.108 \pm 0.024$ \\
\hline Trabecular number $(1 / \mathrm{mm})$ & $1.91(1.71,2.12)$ & $1.67 \pm 0.24$ \\
\hline Trabecular thickness (mm) & $0.066(0.060,0.074)$ & $0.061(0.057,0.072)$ \\
\hline Trabecular separation (mm) & $0.452(0.407,0.520)$ & $0.546 \pm 0.093$ \\
\hline Cortical thickness (mm) & $0.77 \pm 0.18$ & $0.61 \pm 0.08$ \\
\hline \multicolumn{3}{|l|}{ Estimates of bone strength at radius } \\
\hline Stiffness (kN/mm) & $71.1 \pm 15.8$ & $60.0 \pm 2.6$ \\
\hline Failure load (kN) & $3.6 \pm 0.8$ & $2.9 \pm 0.2$ \\
\hline \multicolumn{3}{|l|}{ Microarchitecture parameters at tibia } \\
\hline Trabecular BV/TV (\%) & $0.142 \pm 0.038$ & $0.115 \pm 0.028$ \\
\hline Trabecular number $(1 / \mathrm{mm})$ & $1.87 \pm 0.42$ & $1.70(1.49,1.81)$ \\
\hline Trabecular thickness (mm) & $0.076 \pm 0.013$ & $0.071 \pm 0.008$ \\
\hline Trabecular separation (mm) & $0.458(0.398,0.540)$ & $0.510(0.483,0.638)$ \\
\hline Cortical thickness (mm) & $1.17(0.98,1.33)$ & $0.97 \pm 0.22$ \\
\hline \multicolumn{3}{|l|}{ Estimates of bone strength at tibia } \\
\hline Stiffness (kN/mm) & $200.4 \pm 44.9$ & $165.6 \pm 23.9$ \\
\hline Failure load $(\mathrm{kN})$ & $10.1 \pm 2.2$ & $8.2 \pm 1.3$ \\
\hline \multicolumn{3}{|l|}{ Hematologic parameters } \\
\hline White blood cell count $(\times 10 \mathrm{E} 3 / \mu \mathrm{L})$ & $5.6(4.7,7.1)$ & $4.4 \pm 1.0$ \\
\hline Red blood cell count $(\times 10 \mathrm{E} 6 / \mu \mathrm{L})$ & $4.30 \pm 0.34$ & $4.21 \pm 0.42$ \\
\hline Hemoglobin (g/dL) & $12.9 \pm 1.0$ & $13.1 \pm 1.0$ \\
\hline Hematocrit (\%) & $38.3 \pm 2.7$ & $38.9 \pm 3.2$ \\
\hline Platelet count $(\times 10 \mathrm{E} 3 / \mu \mathrm{L})$ & $254 \pm 60$ & $247 \pm 63$ \\
\hline
\end{tabular}

Values are mean $\pm S D$, or median (interquartile range) when data were not normally distributed. $B M D=$ bone mineral density.

hematocrit level (Fig. 3C and D). There were no significant associations between change in WBC count and change in BMAT.

\section{Discussion}

In this exploratory analysis, we have shown that BMAT, a component of the bone marrow microenvironment, is inversely associated with RBC and WBC counts in a population of premenopausal women. Although the function of BMAT is not known, it is inversely associated with bone parameters and therefore may have a role in mineral metabolism. We now show that there may be an association between BMAT and hematopoietic cells in humans, suggesting a possible role for BMAT in hematopoiesis.

Although BMAT, a component of the bone marrow microenvironment, has been associated with low bone density, ${ }^{(6-8)}$ its function has not been fully described. Paradoxically, in models of chronic starvation, such as anorexia nervosa, although subcutaneous and visceral adipose tissue stores are reduced, levels of BMAT are elevated. ${ }^{(8)}$ Why lipid would be stored in the marrow while other adipose tissue depots are actively being utilized as an energy source during periods of caloric deprivation is not known, but understanding this paradox may allow us to better understand the function of this fat depot.

Given the inverse association between marrow adiposity and bone density, BMAT has been hypothesized to be an important determinant of the low BMD and increased fracture risk observed in women with anorexia nervosa. ${ }^{(26)}$ BMAT is associated with decreased bone integrity ${ }^{(27)}$ and BMAT is inversely associated with finite element analysis-derived estimates of bone strength both in adolescents with anorexia nervosa ${ }^{(28)}$ and, as our data here show, also in premenopausal adult women, but whether BMAT is a causative factor of the increased fracture risk observed in anorexia nervosa remains to be determined.

In addition to marrow adipocytes, the bone marrow microenvironment also consists of osteoblast and hematopoietic 
Table 2. Univariate Associations Between BMAT at the Spine and Hip and Bone Parameters in Cross-Sectional Study Participants ( $n=89$ )

\begin{tabular}{lcccc}
\hline & BMAT at $L_{4}$ & BMAT at femoral & BMAT at femoral & BMAT at femoral \\
vertebra & epiphysis & diaphysis & metaphysis \\
(lipid/water) & (lipid/water) & (lipid/water) & (lipid/water) \\
\hline
\end{tabular}

BMD

Posterior-anterior spine BMD $\left(\mathrm{g} / \mathrm{cm}^{2}\right)$

Lateral spine BMD $\left(\mathrm{g} / \mathrm{cm}^{2}\right)$

Total hip BMD $\left(\mathrm{g} / \mathrm{cm}^{2}\right)$

Femoral neck BMD $\left(\mathrm{g} / \mathrm{cm}^{2}\right)$

Total body BMD $\left(\mathrm{g} / \mathrm{cm}^{2}\right)$

Microarchitecture parameters at radius

Trabecular BV/TV (\%)

Trabecular number $(1 / \mathrm{mm})$

Trabecular thickness $(\mathrm{mm})$

Trabecular separation $(\mathrm{mm})$

Cortical thickness ( $\mathrm{mm}$ )

Estimates of bone strength at radius

Stiffness $(\mathrm{kN} / \mathrm{mm})$

Failure load (kN)

Microarchitecture parameters at tibia

Trabecular BV/TV (\%)

Trabecular number $(1 / \mathrm{mm})$

Trabecular thickness ( $\mathrm{mm}$ )

Trabecular separation $(\mathrm{mm})$

Cortical thickness (mm)

Estimates of bone strength at tibia

Stiffness $(\mathrm{kN} / \mathrm{mm})$

Failure load (kN)

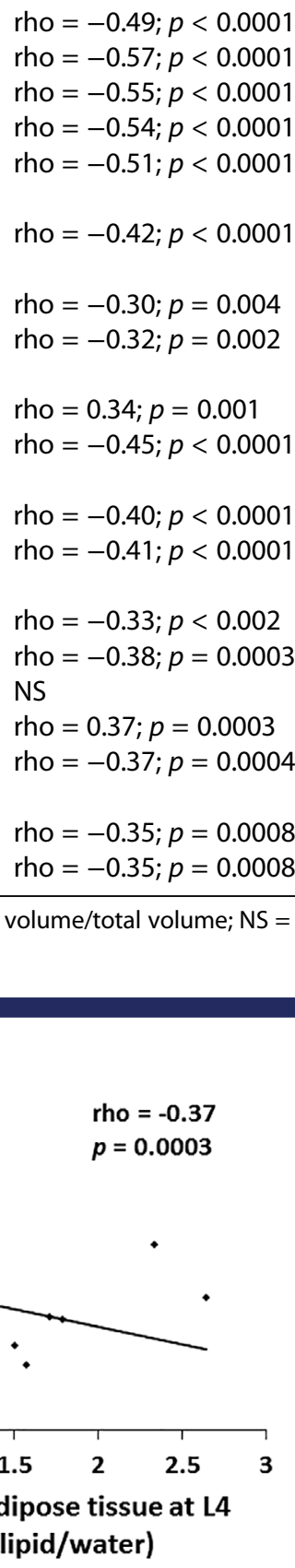

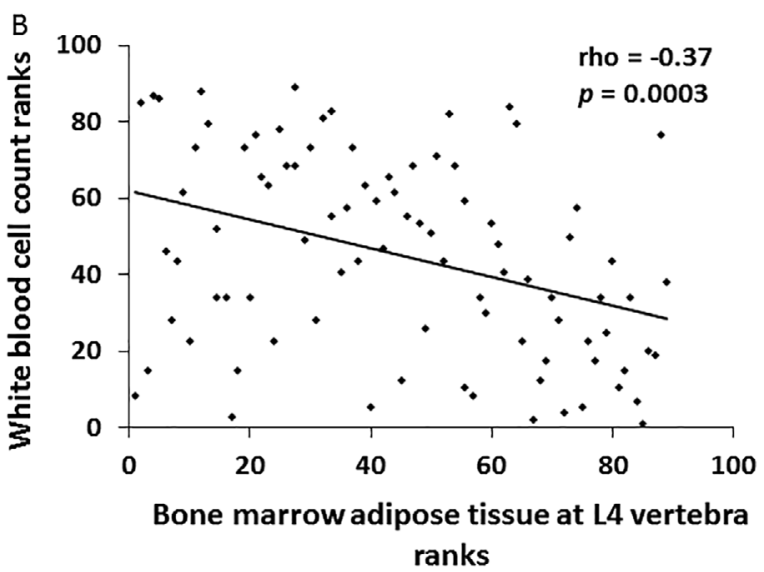

\begin{tabular}{|c|c|c|}
\hline NS & rho $=-0.41 ; p<0.0001$ & rho $=-0.60 ; p<0.0001$ \\
\hline NS & rho $=-0.31 ; p<0.004$ & rho $=-0.62 ; p<0.0001$ \\
\hline NS & rho $=-0.40 ; p=0.0001$ & rho $=-0.63 ; p<0.0001$ \\
\hline NS & rho $=-0.40 ; p<0.0001$ & rho $=-0.63 ; p<0.0001$ \\
\hline NS & rho $=-0.34 ; p=0.001$ & rho $=-0.52 ; p<0.0001$ \\
\hline $\begin{array}{l}=-0.27 \\
p=0.01\end{array}$ & rho $=-0.31 ; p<0.004$ & rho $=-0.50 ; p<0.0001$ \\
\hline $\begin{array}{l}\text { NS } \\
=-0.23 ; \\
p=0.03\end{array}$ & $\begin{array}{l}\text { rho }=-0.23 ; p=0.03 \\
\text { rho }=-0.22 ; p=.04\end{array}$ & $\begin{array}{l}\text { rho }=-0.43 ; p<0.0001 \\
\text { rho }=-0.30 ; p<0.005\end{array}$ \\
\hline NS & rho $=0.27 ; p=0.01$ & rho $=0.47 ; p<0.0001$ \\
\hline NS & rho $=-0.35 ; p=0.0009$ & rho $=-0.40 ; p=0.0001$ \\
\hline NS & NS & rho $=-0.42 ; p<0.0001$ \\
\hline NS & NS & rho $=-0.44 ; p<0.0001$ \\
\hline NS & NS & rho $=-0.37 ; p=0.0003$ \\
\hline NS & rho $=-0.33 ; p<0.002$ & rho $=-0.49 ; p<0.0001$ \\
\hline NS & NS & NS \\
\hline NS & rho $=0.31 ; p<0.003$ & rho $=0.49 ; p<0.0001$ \\
\hline NS & rho $=-0.33 ; p<0.002$ & rho $=-0.42 ; p<0.00$ \\
\hline
\end{tabular}

NS NS

NS NS rho $=-0.39 ; p=0.0002$ rho $=-0.39 ; p=0.0001$

$\mathrm{BMD}=$ bone mineral density; BV/TV = bone volume/total volume; NS = not significant.

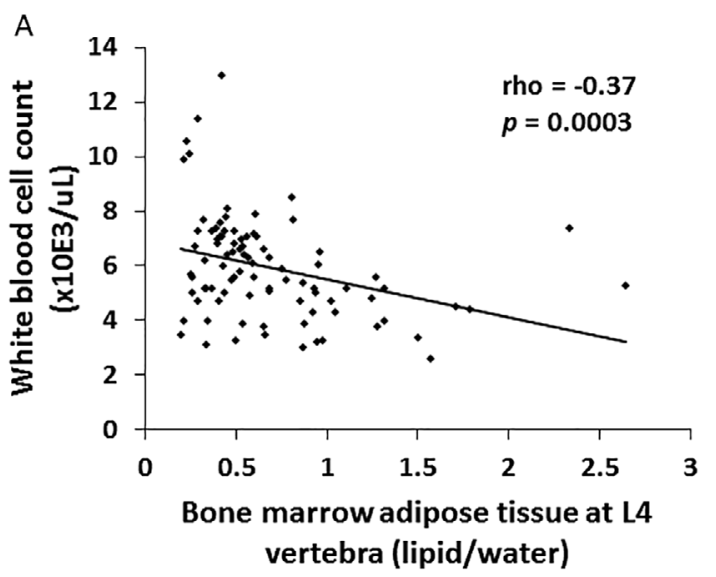

Fig. 1. There was a significant inverse association between white blood cell count and bone marrow adipose tissue at the $L_{4}$ vertebra (rho $=-0.37$, $p=0.0003)(A)$. When we performed a sensitivity analysis and excluded the two subjects with $L_{4} B M A T \geq 2$ lipid/water and the five subjects with WBC count $\geq 9 \times 10 \mathrm{E} 3 / \mu \mathrm{L}$, the association remained significant (rho $=-0.33, p=0.002)$. Data are also plotted as ranks $(B)$.

progenitors. A number of studies in both animal models and humans have investigated the association between hematopoiesis and bone formation. In murine models, osteoblastic cells have been shown to be potential important regulators of hematopoiesis. ${ }^{(1,2)}$ In addition, in human studies, bone loss at the hip has been associated with an increased risk of anemia ${ }^{(5)}$ and hemoglobin levels have been positively associated with BMD measured by $\mathrm{pQCT}{ }^{(4)}$ and ultrasound. ${ }^{(29)}$ Anemia has also been associated with increased fracture risk. ${ }^{(3)}$ Our data demonstrate that the association between RBC and WBC counts and bone parameters is also significant and positive in premenopausal women; both RBC and WBC counts were significantly associated with BMD as well as parameters of bone microarchitecture and estimates of bone strength. 
Table 3. Univariate Associations Between BMAT at the Spine and Hip and Hematopoietic Parameters

\begin{tabular}{|c|c|c|c|c|c|}
\hline & $\begin{array}{l}\text { White blood } \\
\text { cell count }(\times 10 \mathrm{E} 3 / \mu \mathrm{L})\end{array}$ & $\begin{array}{c}\text { Red blood } \\
\text { cell count }(\times 10 \mathrm{E} 6 / \mu \mathrm{L})\end{array}$ & $\begin{array}{l}\text { Hemoglobin } \\
\qquad(\mathrm{g} / \mathrm{dL})\end{array}$ & $\begin{array}{l}\text { Hematocrit } \\
(\%)\end{array}$ & $\begin{array}{l}\text { Platelet count } \\
(\times 10 \mathrm{E} 3 / \mu \mathrm{L})\end{array}$ \\
\hline $\begin{array}{l}\text { BMAT at } \mathrm{L}_{4} \text { vertebra } \\
\quad \text { (lipid/water) }\end{array}$ & $\begin{array}{r}\text { rho }=-0.37 \\
p=0.0003\end{array}$ & NS & NS & NS & NS \\
\hline $\begin{array}{l}\text { BMAT at femoral epiphysis } \\
\text { (lipid/water) }\end{array}$ & NS & NS & NS & NS & NS \\
\hline $\begin{array}{l}\text { BMAT at femoral diaphysis } \\
\text { (lipid/water) }\end{array}$ & $\begin{array}{c}\text { rho }=-0.24 \\
p<0.03\end{array}$ & $\begin{aligned} \text { rho } & =-0.31 \\
p & <0.004\end{aligned}$ & $\begin{aligned} \text { rho } & =-0.20 \\
p & =0.057\end{aligned}$ & $\begin{aligned} \text { rho } & =-0.20 \\
p & =0.057\end{aligned}$ & NS \\
\hline $\begin{array}{l}\text { BMAT at femoral metaphysis } \\
\text { (lipid/water) }\end{array}$ & $\begin{array}{r}\text { rho }=-0.36 \\
p=0.0005\end{array}$ & $\begin{aligned} \text { rho } & =-0.32 \\
p & =0.002\end{aligned}$ & NS & NS & NS \\
\hline
\end{tabular}

NS = not significant

Table 4. Univariate Associations Between BMD, Bone Microarchitecture, Estimated Bone Strength, and Hematopoietic Parameters

\begin{tabular}{|c|c|c|}
\hline Parameter & White blood cell count $(\times 10 \mathrm{E} 3 / \mu \mathrm{L})$ & Red blood cell count $(\times 10 \mathrm{E} 6 / \mu \mathrm{L})$ \\
\hline \multicolumn{3}{|l|}{ BMD } \\
\hline Posterior-anterior spine BMD $\left(\mathrm{g} / \mathrm{cm}^{2}\right)$ & rho $=0.45 ; p<0.0001$ & rho $=0.32 ; p=0.002$ \\
\hline Lateral spine BMD $\left(\mathrm{g} / \mathrm{cm}^{2}\right)$ & rho $=0.52 ; p<0.0001$ & $R=0.29 ; p=0.006$ \\
\hline Total Hip BMD $\left(\mathrm{g} / \mathrm{cm}^{2}\right)$ & rho $=0.49 ; p<0.0001$ & $R=0.30 ; p=0.004$ \\
\hline Femoral neck BMD $\left(\mathrm{g} / \mathrm{cm}^{2}\right)$ & rho $=0.50 ; p<0.0001$ & $R=0.32 ; p=0.002$ \\
\hline Total body BMD $\left(\mathrm{g} / \mathrm{cm}^{2}\right)$ & rho $=0.34 ; p=0.001$ & NS \\
\hline \multicolumn{3}{|l|}{ Radial microarchitecture parameters } \\
\hline Trabecular BV/TV (\%) & rho $=0.33 ; p=0.002$ & rho $=0.25 ; p<0.02$ \\
\hline Trabecular number (1/mm) & rho $=0.26 ; p=0.01$ & rho $=0.29 ; p<0.006$ \\
\hline Trabecular thickness (mm) & rho $=0.25 ; p<0.02$ & NS \\
\hline Trabecular separation (mm) & rho $=-0.29 ; p<0.01$ & rho $=-0.29 ; p<0.007$ \\
\hline Cortical thickness (mm) & rho $=0.24 ; p<0.03$ & NS \\
\hline \multicolumn{3}{|l|}{ Estimates of bone strength at radius } \\
\hline Stiffness (kN/mm) & rho $=0.39 ; p=0.0002$ & $R=0.25 ; p=0.02$ \\
\hline Failure load (kN) & rho $=0.40 ; p<0.0001$ & $R=0.26 ; p=0.01$ \\
\hline \multicolumn{3}{|l|}{ Microarchitecture parameters at tibia } \\
\hline Trabecular BV/TV (\%) & rho $=0.31 ; p=0.003$ & $R=0.28 ; p=0.007$ \\
\hline Trabecular number (1/mm) & rho $=0.38 ; p=0.0003$ & $R=0.26 ; p<0.02$ \\
\hline Trabecular thickness (mm) & NS & NS \\
\hline Trabecular separation (mm) & rho $=-0.37 ; p=0.0004$ & rho $=-0.29 ; p<0.007$ \\
\hline Cortical thickness (mm) & rho $=0.24 ; p=0.02$ & NS \\
\hline \multicolumn{3}{|l|}{ Estimates of bone strength at tibia } \\
\hline Stiffness (kN/mm) & rho $=0.40 ; p=0.0001$ & $R=0.24 ; p<0.03$ \\
\hline Failure load (kN) & rho $=0.41 ; p<0.0001$ & $R=0.24 ; p<0.03$ \\
\hline
\end{tabular}

$\mathrm{BMD}=$ bone mineral density; $\mathrm{BV} / \mathrm{TV}=$ bone volume/total volume; NS = not significant.

In contrast, less is known about the association between marrow adipocytes and hematopoiesis. In a murine model, skeletal regions with greater BMAT have been associated with fewer hematopoietic stem cells and in mice who are irradiated and receive a bone-marrow transplant, inhibition of adipogenesis after irradiation is associated with improved bone-marrow engraftment and increased WBC counts. ${ }^{(10)}$ More recently, reductions in BMAT in a bariatric surgery mouse model have been associated with increased granulocyte-colony stimulating factor which, in turn, leads to increased circulating neutrophils. ${ }^{(9)}$ Our data now show an inverse association between BMAT and both RBC and WBC counts in humans. Whether BMAT is a negative regulator of hematopoiesis ${ }^{(10)}$ or whether it may be utilized to fuel myelopoiesis ${ }^{(9)}$ in humans remains unknown, and future studies will be necessary to further explore this association. Given the known association between BMAT and decreased bone integrity, ${ }^{(27)}$ whether this association between BMAT and hematopoietic parameters is a potential mediator in the association between anemia and increased fracture risk, which has been shown in some populations to be independent of $\mathrm{BMD}^{(30)}$ also warrants further study.

Interestingly, when we divided the group by $\mathrm{BMI}$, we found that the association between BMAT and WBC and RBC counts in overweight/obese women was opposite to that observed in the group as a whole. In overweight/obese women, the relationship between BMAT and WBC/RBC counts was positive. These data are consistent with prior studies suggesting potential differences in BMAT in women with anorexia nervosa as compared to those who are normalweight or overweight/obese. For example, in overweight/ obese women, BMAT is positively associated with visceral adipose tissue, ${ }^{(31)}$ whereas this association is not observed in lower-weight women. ${ }^{(14)}$ These data may provide further support for the concept that BMAT may have a different function in states of nutrient insufficiency as compared to nutrient sufficiency. 

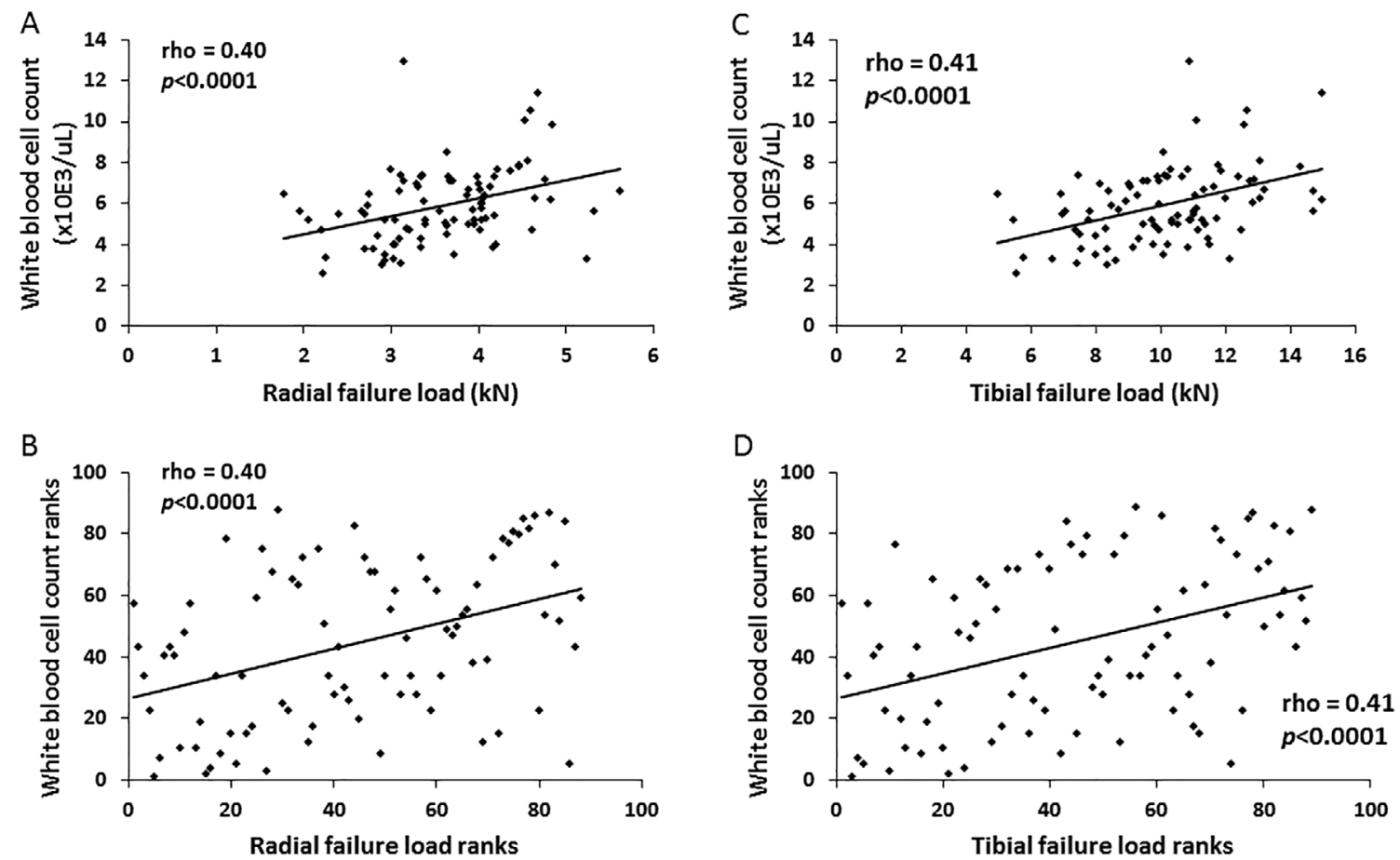

Fig. 2. There was a significant positive association between white blood cell count and failure load, an estimate of bone strength, at the radius ( $r$ ho $=0.40$, $p<0.0001)(A$, with ranks plotted in $B)$, and at the tibia (rho $=0.41, p<0.0001)(C$, with ranks plotted in $D)$.

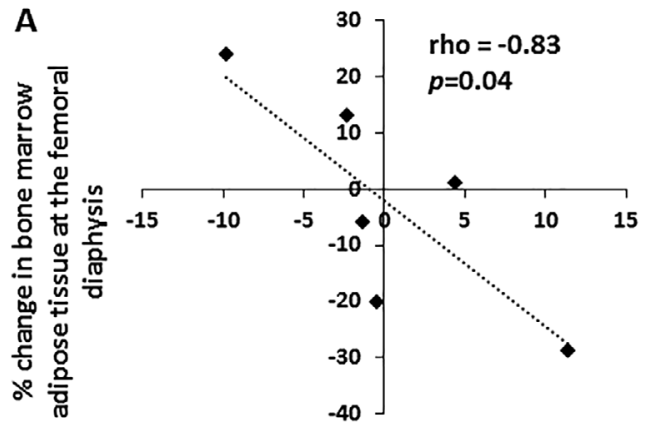

$\%$ change in red blood cell count

B

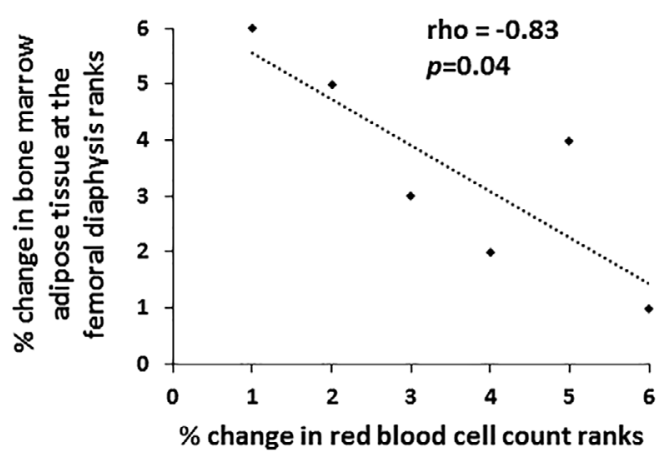

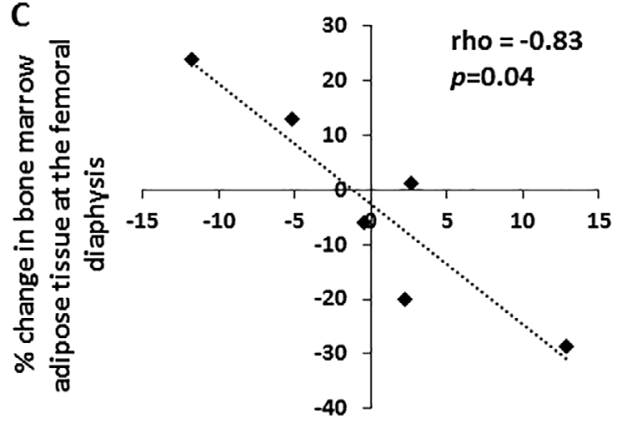

$\%$ change in hematocrit

D

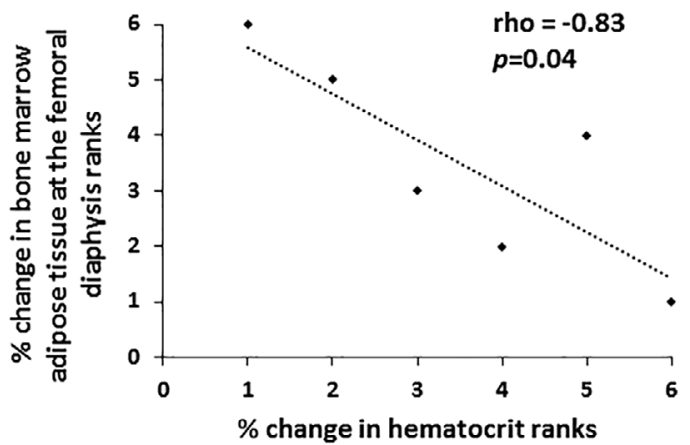

Fig. 3. There was a significant inverse association between $\%$ change in bone marrow adipose tissue at the femoral diaphysis and $\%$ change in red blood cell count ( $\mathrm{rho}=-0.83, p=0.04)(A$, with ranks plotted in $B)$ and $\%$ change in hematocrit (rho $=-0.83, p=0.04)(C$, with ranks plotted in $D)$ after 6 months of transdermal estrogen treatment in six women with anorexia nervosa. 
Although a major limitation of our study is its cross-sectional nature, which does not allow us to make any inferences about causality, the longitudinal component of our study shows a strong association between changes in BMAT and changes in RBC counts over a short-time interval. Importantly, this is a small, exploratory study and therefore meant to be hypothesis-generating, given the very small sample size, but these data suggest that BMAT may potentially be a determinant of RBC count. Estrogen has been shown to increase hematopoietic stem cell division and therefore it will be important to determine in future studies whether this association between changes in BMAT and changes in RBC count is independent of estrogen. ${ }^{(32)}$ An additional limitation is that we did not have data on specific WBC populations (lymphocytes and neutrophil counts) and prior human studies have demonstrated differential findings with respect to the association between BMD and specific WBC populations. ${ }^{(5)}$ Furthermore, the univariate associations we observed in this study are prone to confounding, including potential confounding by inflammatory cytokines. Inflammatory cytokines, specifically interleukin6 (IL-6), have been shown to be associated with low hemoglobin levels in frail, elderly adults ${ }^{(33)}$ and IL-6 is also a mediator of osteoclastogenesis, ${ }^{(34)}$ suggesting a possible link between anemia and decreased BMD. Although our population included premenopausal women, increased IL- 6 levels have been reported both in women with anorexia nervosa and obese individuals $^{(35,36)}$ and therefore future studies will be necessary to further explore the possible association between inflammatory cytokines, BMD, and blood counts in these populations.

In conclusion, in this exploratory study, we show an association between BMAT and RBC and WBC counts. Further studies will therefore be necessary to better understand the complex relationship between adipocyte, osteoblast, and hematopoietic precursors in the marrow microenvironment in order to gain insight into the function of BMAT.

\section{Disclosures}

All authors state that they have no conflicts of interest.

\section{Acknowledgments}

This work was supported by grants UL 1TR002541 (The Harvard Clinical and Translational Science Center, National Center for Advancing Translational Sciences); UL1 RR025758 (Harvard Clinical and Translational Science Center); 1S10RR023405 (National Center for Research Resources); and NIH grants R24 DK084970, R03 DK084970, and P30 DK040561. The content is solely the responsibility of the authors and does not necessarily represent the official views of the National Institutes of Health. We would like to thank the nurses and bionutritionists of the MGH Clinical Research Center for their expert care.

Authors' roles: Study design of original studies: PKF, AK, and OAM. Study conduct: SP, MR, ATF, EM, and PKF. Data collection/ analysis: SP, MR, ATF, EM, MAB, MB, and PKF. Statistical analysis: PKF and HL. Drafting manuscript: PKF. Revising manuscript content: All authors. Approving final version of manuscript: All authors. PKF takes responsibility for the integrity of the data analysis.

\section{References}

1. Calvi LM, Adams GB, Weibrecht KW, et al. Osteoblastic cells regulate the haematopoietic stem cell niche. Nature. 2003;425(6960):841-6.

2. Visnjic D, Kalajzic Z, Rowe DW, Katavic V, Lorenzo J, Aguila HL. Hematopoiesis is severely altered in mice with an induced osteoblast deficiency. Blood. 2004;103(9):3258-64.

3. van Staa TP, Leufkens HG, Cooper C. Utility of medical and drug history in fracture risk prediction among men and women. Bone. 2002; 31(4):508-14.

4. Cesari M, Pahor M, Lauretani F, et al. Bone density and hemoglobin levels in older persons: results from the InCHIANTI study. Osteoporos Int. 2005;16(6):691-9.

5. Valderrabano RJ, Lui LY, Lee J, et al. Bone density loss is associated with blood cell counts. J Bone Miner Res. 2017;32(2):212-20.

6. Shen W, Chen J, Punyanitya M, Shapses S, Heshka S, Heymsfield SB. MRI-measured bone marrow adipose tissue is inversely related to DXA-measured bone mineral in Caucasian women. Osteoporos Int. 2007;18(5):641-7.

7. Shen $W$, Scherzer R, Gantz M, et al. Relationship between MRImeasured bone marrow adipose tissue and hip and spine bone mineral density in African-American and Caucasian participants: the CARDIA study. J Clin Endocrinol Metab. 2012;97(4):1337-46.

8. Bredella MA, Fazeli PK, Miller KK, et al. Increased bone marrow fat in anorexia nervosa. J Clin Endocrinol Metab. 2009;94(6):2129-36.

9. Li Z, Hardij J, Evers SS, et al. G-CSF partially mediates effects of sleeve gastrectomy on the bone marrow niche. J Clin Invest. 2019;130: 2404-16.

10. Naveiras O, Nardi V, Wenzel PL, Hauschka PV, Fahey F, Daley GQ. Bone-marrow adipocytes as negative regulators of the haematopoietic microenvironment. Nature. 2009;460(7252):259-63.

11. Bredella MA, Fazeli PK, Daley SM, et al. Marrow fat composition in anorexia nervosa. Bone. 2014;66:199-204.

12. Cawthorn WP, Scheller EL, Learman BS, et al. Bone marrow adipose tissue is an endocrine organ that contributes to increased circulating adiponectin during caloric restriction. Cell Metab. 2014;20(2):368-75.

13. Fazeli PK, Faje AT, Cross EJ, et al. Serum FGF-21 levels are associated with worsened radial trabecular bone microarchitecture and decreased radial bone strength in women with anorexia nervosa. Bone. 2015;77:6-11.

14. Fazeli PK, Faje A, Bredella MA, et al. Changes in marrow adipose tissue with short-term changes in weight in premenopausal women with anorexia nervosa. Eur J Endocrinol. 2019;180(3):189-99.

15. Resulaj M, Polineni S, Meenaghan E, Eddy KT, Lee H, Fazeli PK. Transdermal estrogen in women with anorexia nervosa: an exploratory pilot study. JBMR Plus. 2020;4(1):e10251.

16. Syed FA, Oursler MJ, Hefferanm TE, Peterson JM, Riggs BL, Khosla S. Effects of estrogen therapy on bone marrow adipocytes in postmenopausal osteoporotic women. Osteoporos Int. 2008;19(9):1323-30.

17. Ettinger $B$, Ensrud KE, Wallace $R$, et al. Effects of ultralow-dose transdermal estradiol on bone mineral density: a randomized clinical trial. Obstet Gynecol. 2004;104(3):443-51.

18. Schorr M, Fazeli PK, Bachmann KN, et al. Differences in trabecular plate and rod structure in premenopausal women across the weight spectrum. J Clin Endocrinol Metab. 2019;104(10):4501-10.

19. American Psychiatric Association. Diagnostic and Statistical Manual of Mental Disorders (DSM-5). 5th ed. Washington, DC: American Psychiatric Association; 2013.

20. Johnson J, Dawson-Hughes B. Precision and stability of dual-energy X-ray absorptiometry measurements. Calcif Tissue Int. 1991;49(3): $174-8$.

21. Boutroy S, Bouxsein ML, Munoz F, Delmas PD. In vivo assessment of trabecular bone microarchitecture by high-resolution peripheral quantitative computed tomography. J Clin Endocrinol Metab. 2005; 90(12):6508-15.

22. Pistoia W, van Rietbergen B, Lochmuller EM, Lill CA, Eckstein F, Ruegsegger P. Estimation of distal radius failure load with microfinite element analysis models based on three-dimensional 
peripheral quantitative computed tomography images. Bone. 2002; 30(6):842-8.

23. Macneil JA, Boyd SK. Bone strength at the distal radius can be estimated from high-resolution peripheral quantitative computed tomography and the finite element method. Bone. 2008;42(6): 1203-13.

24. Mueller TL, Christen D, Sandercott S, et al. Computational finite element bone mechanics accurately predicts mechanical competence in the human radius of an elderly population. Bone. 2011;48(6): 1232-8.

25. Fazeli PK, Bredella MA, Freedman L, et al. Marrow fat and preadipocyte factor-1 levels decrease with recovery in women with anorexia nervosa. J Bone Miner Res. 2012;27(9):1864-71.

26. Miller KK, Grinspoon SK, Ciampa J, Hier J, Herzog D, Klibanski A. Medical findings in outpatients with anorexia nervosa. Arch Intern Med. 2005;165(5):561-6.

27. Schellinger D, Lin CS, Hatipoglu HG, Fertikh D. Potential value of vertebral proton MR spectroscopy in determining bone weakness. AJNR Am J Neuroradiol. 2001;22(8):1620-7.

28. Singhal V, Tulsiani S, Campoverde KJ, et al. Impaired bone strength estimates at the distal tibia and its determinants in adolescents with anorexia nervosa. Bone. 2018;106:61-8.

29. Laudisio A, Marzetti E, Pagano F, Bernabei R, Zuccala G. Haemoglobin levels are associated with bone mineral density in the elderly: a population-based study. Clin Rheumatol. 2009;28(2): 145-51.

30. Valderrabano RJ, Lee J, Lui LY, et al. Older men with anemia have increased fracture risk independent of bone mineral density. J Clin Endocrinol Metab. 2017;102(7):2199-206.

31. Bredella MA, Torriani M, Ghomi RH, et al. Vertebral bone marrow fat is positively associated with visceral fat and inversely associated with IGF-1 in obese women. Obesity (Silver Spring). 2011;19(1):49-53.

32. Nakada D, Oguro $H$, Levi BP, et al. Oestrogen increases haematopoietic stem-cell self-renewal in females and during pregnancy. Nature. 2014;505(7484):555-8.

33. Leng S, Chaves $P$, Koenig $K$, Walston J. Serum interleukin- 6 and hemoglobin as physiological correlates in the geriatric syndrome of frailty: a pilot study. J Am Geriatr Soc. 2002;50(7):1268-71.

34. Jilka RL, Hangoc G, Girasole G, et al. Increased osteoclast development after estrogen loss: mediation by interleukin-6. Science. 1992; 257(5066):88-91.

35. Solmi M, Veronese N, Favaro A, et al. Inflammatory cytokines and anorexia nervosa: a meta-analysis of cross-sectional and longitudinal studies. Psychoneuroendocrinology. 2015;51:237-52.

36. Kern PA, Ranganathan S, Li C, Wood L, Ranganathan G. Adipose tissue tumor necrosis factor and interleukin- 6 expression in human obesity and insulin resistance. Am J Physiol Endocrinol Metab. 2001;280(5): E745-51. 